time was less than 3 weeks and only one-fifth of patients required nocturnal non-invasive ventilation. NMD-CWD patients were most likely to survive hospital but almost one-third of COPD patients died during the weaning process. COPD patients had the worst outcome at 2-years, whereas over half of the NMD-CWD patients were alive at 2-years. These data strongly support the Department of Health's plan to develop 'Complex Home Ventilation, Weaning and Rehabilitation Centres' as part of the investment in specialist respiratory services to enhance patient outcome.

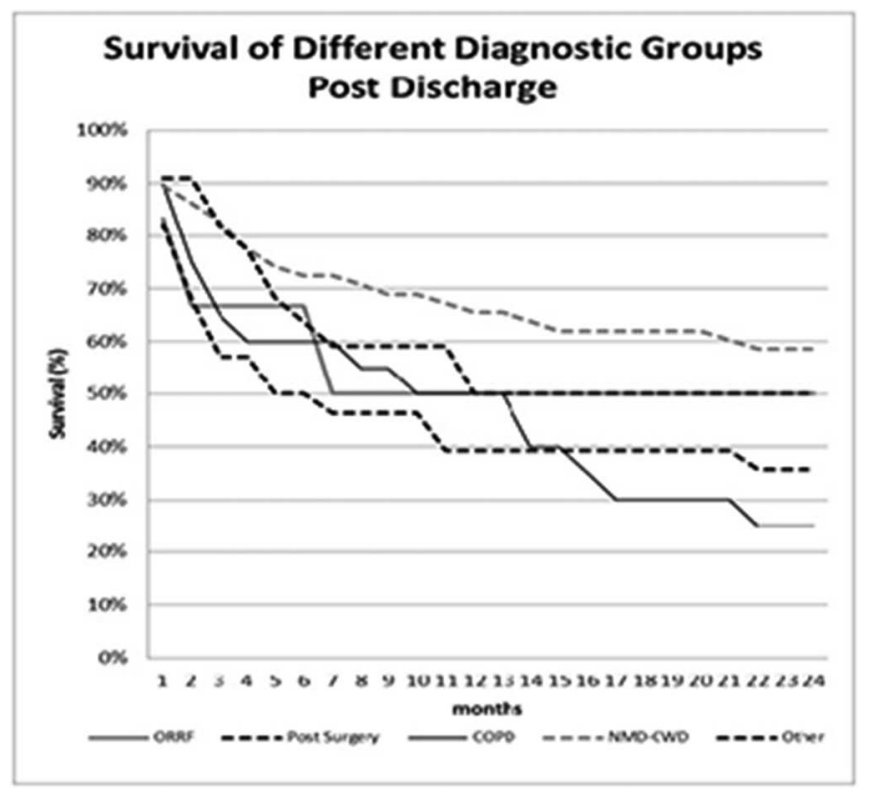

Abstract S133 Figure 1

\section{S134 ACTIVITY MONITORING IN INTENSIVE CARE UNIT SURVIVORS: ASSESSING DAILY PHYSICAL ACTIVITY WITH OBJECTIVE OUTCOME MEASURES}

${ }^{1}$ AS McNelly, ${ }^{1}$ J Rawal, ${ }^{1} \mathrm{P}$ Chan, ${ }^{2} \mathrm{~N}$ Hopkinson, ${ }^{3} \mathrm{~J}$ Moxham , ${ }^{4}$ SDR Harridge, ${ }^{5} \mathrm{~N}$ Hart, ${ }^{1} \mathrm{H}$ Montgomery, ' $\mathrm{Z}$ Puthucheary; 'Institute of Health and Human Performance, UCL, London, UK; ${ }^{2}$ Royal Brompton Hospital, Imperial College, London, UK; ${ }^{3}$ King's College Hospital NHS Foundation Trust, London, UK; ${ }^{4}$ Centre of Human and Aerospace Physiological Sciences, King's College London, London, UK; ${ }^{5}$ Lane Fox Clinical Respiratory Physiology Unit, Guy's and St Thomas' NHS Foundation Trust, London, UK

10.1136/thoraxjnl-2013-204457.141

Introduction and Objectives Intensive care unit (ICU) survivors commonly report long-term functional disability and reduced daily physical activity (PA). This adversely impacts on their health-related quality of life (HRQL). We aimed to investigate the relationship between subjective and objective measures of PA in this patient group.

Methods Subjects were ICU survivors drawn from the 63 participants of the Musculoskeletal Ultrasound Study in Critical Care: Longitudinal Evaluation (MUSCLE) study. All were over 16 years, invasively ventilated for $>48$ hours with an ICU length of stay $>7$ days. Objective (SenseWear Pro accelerometers, BodyMedia Inc, Pittsburgh, US) and self-reported subjective PA levels (HRQL SF-36 questionnaire, QualityMetric Inc, Lincoln, US) were measured.

Results At 18 months 20 pts had died; 17 were lost to followup, and 1 withdrew. 27 patients were studied (14 female) with a mean age \pm SEM of $56.6 \pm 3.6$ years and a post-ICU discharge time of $576 \pm 190 \mathrm{~d}$. SF-36 scores were lower than normal range scores. Age-adjusted (AA) mean steps/day correlated with norm-based physical component summary (PCS) SF-36 scores (Pearson's $\mathrm{r}=+0.58$; $<<0.01$ ), but there was no correlation with the mental component summary scores (Fig. 1). AA daily step variation showed positive associations with absolute levels of AA daily steps $(\mathrm{r}=+0.85 ; \mathrm{p}<0.01)$, SF-36 physical function $(\mathrm{PF})$ scores $(\mathrm{r}=+0.62 ; \mathrm{p}<0.01)$, and prior working status $(\mathrm{r}=+0.69 ; \mathrm{p}<0.01) ;$ and a negative association with chronic disease $(r=-0.54 ; p<0.01)$. Levels of AA daily steps contributed more to AA daily step variation than SF scores $\left(\mathrm{r}^{2}=0.72\right.$, 0.38 respectively). Receiver Operator Characteristic analysis indicated that AA daily steps and PF scores are good (but non-significant) predictors for working post-ICU $(0.88,0.86$ respectively; $\mathrm{p}>0.05$ ).

Conclusion This is the first report on the use of activity monitors in ICU survivors, and indicates that subjective and objective measures of PA are correlated. Such complementary data can be used to investigate functional disability in critical illness survivors. Both methods of assessing PA identified strong associations with health and socio-economic factors. Variation in step count, and specifically lack of variation in step count, may be more attributable to absolute step count than self-reported PF or chronic disease. Objective PA measures may offer advantages for monitoring postICU patients compared with subjective methods.

\section{Correlations between Activity and SF-36 forms}

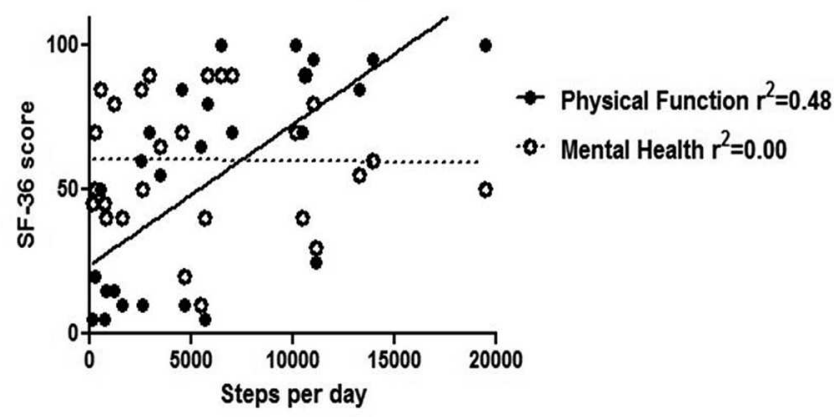

Abstract S134 Figure 1. Correlations between Physical Function and Mental Component Summary SF-36 Scores against Physical Activity.

S135 LONG-TERM OUTCOMES IN PATIENTS REFERRED TO A
SPECIALISED WEANING CENTRE; THE IMPACT OF
REFERRAL SOURCE, NON-INVASIVE VENTILATION AND
DIAGNOSIS

MG Davies, D Horton, R Chadwick, NS Oscroft, TG Quinnell, JM Shneerson, IE Smith; Papworth Hospital NHS Foundation Trust, Cambridge, UK

\subsection{6/thoraxjnl-2013-204457.142}

Introduction The expansion of specialised weaning centres serving networks of external ICUs has been recommended by the NHS commissioning board. However, data are limited for this proposed model of care. Papworth Hospital provides a multi-disciplinary weaning service in-line with these recommendations. Methods We analysed the records of patients transferred to our service for weaning from invasive ventilation (IMV) between 1992-2011 inclusive. Patients were categorised according to diagnosis (neuromuscular, COPD, post-surgical, non-COPD respiratory, chest-wall disorders and others). 